\title{
The role of grain boundaries and their triple junctions in strengthening and softening of nanocrystalline ceramics
}

\author{
A. G. Sheinerman ${ }^{\dagger, 1,2,3}$, M. Yu. Gutkin ${ }^{1,2,4}$ \\ †asheinerman@gmail.com

\begin{abstract}
${ }^{1}$ Institute for Problems in Mechanical Engineering, RAS, 61 Bolshoy Av. V.O., St. Petersburg 199178, Russia ${ }^{3}$ Saint Petersburg State University, 7/9 Universitetskaya Emb., St. Petersburg, 199034, Russia ${ }^{4}$ ITMO University, 49 Kronverksky Av., St. Petersburg, 197101, Russia
\end{abstract} \\ ${ }^{2}$ Peter the Great Saint Petersburg Polytechnic University, 29 Polytechnicheskaya St., St. Petersburg, 195251, Russia
}

\begin{abstract}
We propose a model that describes both direct and inverse Hall-Petch dependences observed in nanocrystalline ceramics as well as the low strain rate sensitivity of such ceramics. Within the model, plastic deformation in nanocrystalline ceramics is realized via the emission of lattice and grain boundary (GB) dislocations from GB steps and triple junctions of GBs. The model assumes that in the beginning of plastic deformation, the applied load linearly scales with plastic strain and that each GB step or triple junction can emit a dislocation no more than once. The model predicts that the transition from the direct to the inverse Hall-Petch dependence is associated with an increase in the number density of triple junctions as grain size decreases. It is demonstrated that the critical grain size for this transition depends on the fraction of triple junctions that can emit lattice or GB dislocation at a given stress. In turn, the intensity of GB dislocation emission from triple junctions can depend on the structure and energy of GBs and their chemical composition. The model explains the experimental observations (D. N. F. Mucho et al.,. Mater. Lett. 186, 298 (2017); C. Yang et al., J. Amer. Cer. Soc. 102, 6904 (2019)) of direct Hall-Petch dependences down to very small grain sizes by assuming that the critical grain size for the transition from the direct to the inverse Hall-Petch dependence for the materials synthesized in these experiments is smaller than the minimum grain size of the fabricated specimens.
\end{abstract}

Keywords: nanocrystalline ceramics, hardness, strength, plasticity, grain boundaries.

The mechanical properties of ceramics depend dramatically on their grain size. In particular, the hardness of ceramics is commonly linearly related to the inverse of the square root of the grain size, following the Hall-Petch relation [1-3]. This implies that like metals, ceramics tend to strengthen as their grain size is reduced. At the same time, in nanocrystalline ceramics, several research groups [4-14] observed an inverse Hall-Petch relation below a critical grain size, which manifests itself in softening (hardness reduction) with a decrease in grain size (see also review [15]). In contrast, recently, the authors of refs. [16,17] produced superhard nanocrystalline $\mathrm{MgAl}_{2} \mathrm{O}_{4}$ and $\mathrm{ZnAl}_{2} \mathrm{O}_{4}$ ceramics, which demonstrated direct Hall-Petch dependences down to the grain size of 7 and $10 \mathrm{~nm}$, respectively. The reason for these contradictions is not clear yet, and their explanation requires the understanding of the plastic deformation processes in nanoceramics.

Several molecular dynamics simulations [10,18,19] demonstrated that the transition from the direct to the inverse Hall-Petch dependence at a critical grain size can be related to the transition from intragrain plasticity to grain boundary (GB) sliding combined with grain rotation [10,16] and sometimes amorphization of GB regions [10,17]. In addition, GB amorphization, GB sliding, grain rotation and shear banding in nanocrystalline ceramics under indentation were directly observed using transmission electron microscopy $[10,14]$.

The inverse Hall-Petch dependence for nanocrystalline ceramics has also been treated within several mechanical models. For example, Jiang and Weng [20] and Ehre and Chaim [6] applied two- or multiphase composite models to nanocrystalline ceramics and obtained an inverse Hall-Petch dependence below some critical grain size, for the situation where the yield strength of the GB phase is chosen to be sufficiently small. These models, however, do not explain the contradictions in the experimental data on the hardness of nanocrystalline $\mathrm{MgAl}_{2} \mathrm{O}_{4}$.

Besides, recently [21], we have employed the concept [22] of thermally activated GB sliding to explain both strengthening and softening of nanocrystalline ceramics. Within model [21], the transition from the direct to the inverse Hall-Petch behavior is associated to the transition from lattice dislocation slip to the thermally activated GB sliding at a critical grain size. This model explained the contradictory data $[8,9,16]$ on the direct and inverse Hall-Petch behavior of nanocrystalline $\mathrm{MgAl}_{2} \mathrm{O}_{4}$. At the same time, this model predicted high values of strain rate sensitivity (around several tenths) typical of GB sliding in metals. Although the presence of the dependence 
of hardness of nanocrystalline ceramics on their strain rate agrees with recent measurements of the compressive yield strength of YSZ (yittria stabilized zirconia) nanopillars [12], it contradicts to the experimental results [9] for nanocrystalline $\mathrm{MgAl}_{2} \mathrm{O}_{4}$, which demonstrate that the hardness of these nanocrystalline ceramics is nearly independent on the strain rate. Therefore, in the present paper, we suggest a model that explains both the contradictive results on grain size dependence of hardness of nanocrystalline $\mathrm{MgAl}_{2} \mathrm{O}_{4}[8,9,16]$ and a low strain rate sensitivity of these nanocrystalline ceramics.

Within our model, consider a polycrystalline ceramic solid under a uniaxial compressive stress $\sigma$. Following [23], assume that at small enough values of plastic strain, plastic deformation occurs via dislocation emission from GBs and their triple junctions. Assume that GBs emit lattice (perfect or partial) dislocations in grain interiors while triple junctions can either emit lattice dislocations to grain interiors or produce GB dislocations that move along GBs (Fig. 1). We further suppose that GBs emit lattice dislocations from GB steps in such a way that each emitted dislocation eliminates a GB step, thus reducing the GB energy (Fig. 1). If the resolved shear stress in the dislocation slip plane is high enough, dislocation emission from a GB step or a triple junction to the grain interior can be followed by dislocation propagation across the grain and its absorption by the opposite GB. Similarly, the emission of a GB dislocation from a triple junction to a GB can be followed by the motion of such a GB dislocation across the GB to the neighboring triple junction.

Since dislocation emission from an atomic height GB step eliminates the step, following [23], we assume that at small enough values of strain, any GB step can emit a lattice dislocation no more than once. Similarly, we assume that dislocation emission from a triple junction significantly reduces the stresses acting near the junction, so that in the examined case of small strains, each triple junction can emit a dislocation no more than once.

We denote the grain size of the ceramic solid as $d$, the magnitude of the lattice and GB dislocation Burgers vectors as $b$ and $b_{G B}$, respectively, the GB length as $d / 2$ the average spacing between GB steps as $p$ (Fig. 1), and the magnitude of

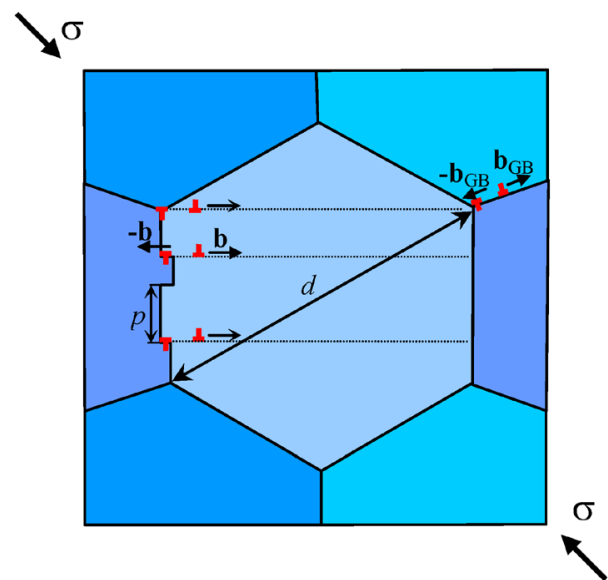

Fig. 1. (Color online) A fragment of a nanocrystalline ceramic solid (under a uniform compressive stress $\sigma$ ) where plastic deformation is realized via dislocation emission from grain boundary steps and triple junctions. the compressive strain as $\varepsilon$. Then the plastic strain $\varepsilon$ can be presented as

$$
\varepsilon \approx \frac{b}{M d}\left(7 N \rho_{s t e p}+12\left(\rho_{t j}^{\text {lat }}+\left(b_{G B} / b\right) \rho_{t j}^{G B}\right)\right) .
$$

In formula (1) $M$ is the Taylor factor [24], $N=d /(2 p)$ is the average number of GB steps per GB, $\rho_{\text {step }}$ and $\rho_{t j}^{\text {lat }}$ is the fraction of steps and triple junctions, respectively, which have emitted lattice dislocations that passed across a grain, $\rho_{t j}^{G B}$ is the fraction of triple junctions that have emitted $\mathrm{GB}$ dislocations that passed across a GB, and the factors 7 and 12 designate the number of GBs and triple junctions, respectively, per grain in the case where all GBs represent the facets of truncated octahedrons. (A truncated octahedron contains 14 facets and 36 edges. Each facet (GB) is shared by 2 adjacent truncated octahedrons, whereas each edge (triple junction) is shared by 3 neighboring truncated octahedrons.)

For convenience, we introduce the denotation $\rho_{t j}=\rho_{t j}^{\text {lat }}+\left(b_{G B} / b\right) \rho_{t j}^{G B}$. Following [23], we assume that at sufficiently small values of strain, the parameters $\rho_{\text {step }}$ and $\rho_{t j}$ linearly scale with the applied load $\sigma$ as

$$
\rho_{s t e p}=\left(\sigma-\sigma_{0}\right) / \sigma_{m 1}, \rho_{t j}=\left(\sigma-\sigma_{0}\right) / \sigma_{m 2},
$$

where $\sigma_{0}, \sigma_{m 1}$ and $\sigma_{m 2}$ are parameters that can depend on grain size and plastic strain. (At $\sigma=\sigma_{0}+\sigma_{m 1}$ or $\sigma=\sigma_{0}+\sigma_{m 2}$, formulae (2) yield: $\rho_{\text {step }}=1$ or $\rho_{t j}=1$, respectively, which means that all GB steps or all triple junctions have emitted a dislocation. This implies that the parameters $\sigma_{m 1}$ and $\sigma_{m 2}$ determine the maximum strengths, $\sigma_{0}+\sigma_{m 1}$ and $\sigma_{0}+\sigma_{m 2}$, of dislocation sources at GB steps and triple junctions, respectively.) Formulae (1) and (2) can be valid in the situation where at small strain, the applied load $\sigma$ linearly scales with plastic strain $\varepsilon$. Such a situation takes place, for example, during compression of some nanocrystalline metals or composites $[25,26]$. Substituting formulae (2) to formula (1), one obtains:

$$
\varepsilon=\frac{b\left(\sigma-\sigma_{0}\right)\left(7 d \sigma_{m 2}+24 p \sigma_{m 1}\right)}{2 M p d \sigma_{m 1} \sigma_{m 2}},
$$

which yields:

$$
\sigma=\sigma_{0}+\frac{2 M \sigma_{m 1} \sigma_{m 2} \varepsilon p d}{b\left(7 d \sigma_{m 2}+24 p \sigma_{m 1}\right)} .
$$

In the limit of a large enough grain size $\left(d / p \gg \rho_{t j} / \rho_{\text {step }}\right)$, when dislocation emission from triple junctions (described by the second term in formula (1)) can be neglected, formula (1) reduces to $\varepsilon=7 b \rho_{\text {step }} / 2 M p$. The substitution of the first formula from (2) to the latter relation yields:

$$
\sigma=\sigma_{0}+\frac{2 M \sigma_{m 1} \varepsilon p}{7 b}
$$

At small values of $\varepsilon$, formula (5) describes the flow stress of a ceramic solid with a large enough grain size. This implies that formula (5) should comply with the Hall-Petch relation (e.g., [27]) $\sigma=\sigma_{0}^{H P}+k_{\varepsilon} d^{-1 / 2}$, where $\sigma_{0}^{H P}$ and $k_{\varepsilon}$ are grain size independent parameters. This can be achieved if we put $\sigma_{0}^{H P}=\sigma_{0}, k_{\varepsilon}=2 M k_{m 1} \varepsilon p /(7 b)$, where $k_{m 1}=\sigma_{m 1} d^{1 / 2}$ is the parameter independent of $d$. Similarly, we assume that the second formula from (2) can be presented as $\rho_{t j}=\left(\sigma-\sigma_{0}\right) d^{-1 / 2} / k_{m 2}$, where $k_{m 2}=\sigma_{m 2} d^{1 / 2}$ is the parameter independent of $d$. 
Substituting the relations $k_{m 1}=\sigma_{m 1} d^{1 / 2}$ and $k_{m 2}=\sigma_{m 2} d^{1 / 2}$ to formula (4), we obtain

$$
\sigma=\sigma_{0}+\frac{2 M k_{m 1} k_{m 2} \varepsilon p}{b\left(7 k_{m 2} d^{1 / 2}+24 p k_{m 1} d^{-1 / 2}\right)} .
$$

Assuming that the yield strength $\sigma_{y}$ corresponds to the case $\varepsilon=\varepsilon_{0}$, where $\varepsilon_{0}=2 \times 10^{-3}$, formula (6) allows one to calculate the yield strength $\sigma_{y}=\left.\sigma\right|_{\varepsilon=\varepsilon_{0}}$ of the ceramic solid and its microhardness, roughly estimated as: $H_{V} \approx 3 \sigma_{\mathrm{v}}$ [28]. As follows from formula (6), at small enough values of the grain size $d$, the second term in the ratio denominator prevails over the first term, and $H_{V}$ increases with an increase in $d$, while at sufficiently large grain size, the first term in the ratio denominator prevails, and $H_{V}$ decreases as the grain size $d$ increases. This means that there exists a critical grain size $d=d_{c}$ at which the dependence of $H_{V}$ on $d$ has a maximum. The critical grain size $d_{c}$ can be found from the relation $\left.\left(\partial H_{V} / \partial d\right)\right|_{d=d_{c}}=0$, formula (6) and the equalities $\sigma_{y}=\left.\sigma\right|_{\varepsilon=\varepsilon_{0}}$ and $H_{V}=3 \sigma_{y}$ as

$$
d_{c}=\frac{12 b k_{H P}}{M \varepsilon_{0} k_{m 2}},
$$

where $k_{H P}=\left.k_{\varepsilon}\right|_{\varepsilon=\varepsilon_{0}}$. From Eq. (7), we have: $k_{m 2}=12 b k_{H P} /\left(M \varepsilon_{0} d_{c}\right)$. Now insertion of the latter equality and the relations $k_{m 1}=7 b k_{H P} /\left(2 M \varepsilon_{0} p\right)$ and $\sigma_{y}=\left.\sigma\right|_{\varepsilon=\varepsilon_{0}}$ to formula (6) gives:

$$
\sigma_{y}=\sigma_{0}+\frac{k_{H P} d^{1 / 2}}{d+d_{c}} \text {. }
$$

At large enough grain sizes, such that $d \gg d_{c}$, formula (8) reduces to the classical Hall-Petch dependence $\sigma_{y}=\sigma_{0}+k_{H P} d^{-1 / 2}$. In contrast, at sufficiently small grain sizes, such that $d \ll d_{c}$, formula (8) can be written as $\sigma_{y}=\sigma_{0}+\left(k_{H P} / d_{c}\right) d^{1 / 2}$.

The critical grain size $d_{c}$ depends on the intensities of dislocation emission from GB steps and triple junctions, which, in turn, can depend on the structure of GBs and the specimen processing history. Indeed, if specimen fabrication has been accompanied by GB sliding, the latter can produce steps at GB junctions, whose presence can promote GB dislocation emission from triple junctions during plastic deformation of the nanoceramic solid. This implies that the fraction $\rho_{t j}$ of triple junctions, emitting dislocations, should increase if GB sliding occurred during specimen fabrication. Since $k_{m 2}$ is inversely proportional to $\rho_{t j}$ (see formula (2) and the relation $k_{m 2}=\sigma_{m 2} d^{1 / 2}$ ) and $d_{c}$ is inversely proportional to $k_{m 2}, d_{c}$ should be directly proportional to $\rho_{t j}$. This means that GB sliding during specimen fabrication should increase the critical grain size $d_{c}$, thereby shifting the region of the inverse Hall-Petch behavior to larger grain sizes and reducing the maximum achievable hardness (characterized by the maximum at the dependence of $H_{V}$ on $d$ ).

Using formula (8), we have calculated the dependences of microhardness $H_{V}$ of $\mathrm{MgAl}_{2} \mathrm{O}_{4}$ on grain size $d$. To do so, we put $\sigma_{0}=4.2 \mathrm{GPa}[2,8,9,16]$ and $k_{H P}=0.72 \mathrm{MPa} \times \mathrm{m}^{1 / 2}$ $[2,8,9,16]$. The dependences of $H_{V}$ on $d$ are presented in Fig. 2, for various values of the critical grain size $d_{c}$. It is seen from Fig. 2 that the calculated values of the hardness of the nanocrystalline $\mathrm{MgAl}_{2} \mathrm{O}_{4}$ spinel agree with experimental data $[2,8,9,16]$, although the slopes of the sections of the curves that correspond to the inverse Hall-Petch dependence are smaller than it could be expected from these experimental results.
Thus, within our model, there always exists a transition from the direct to the inverse Hall-Petch relation at small grain sizes. The critical grain size $d_{c}$ for this transition can depend on the structure and energy of GBs and their chemical composition. Indeed, recent experiments on nanocrystalline YSZ ceramics [11] demonstrated a significant increase in the GB energy with a decrease in grain size below the critical grain size for the transition from the direct to the inverse Hall-Petch dependence for hardness. This situation is similar to that for nanocrystalline metallic alloys where high-energy (nonequilibrium) GBs decrease the yield strength and hardness by promoting GB sliding, while reducing GB energies via lowtemperature annealing increases the strength and hardness of such solids [29-31]. (A similar effect of hardening by annealing has been reported for various ultrafine-grained metals and alloys (e.g., [31-33]) and theoretically described $[33,34]$ for pure ultrafine grained $\mathrm{Al}$ produced by highpressure torsion.) One can, therefore, speculate that in nanocrystalline ceramics, the formation of high-energy GBs also facilitates the emission of GB dislocations from triple junctions or their subsequent slip across GBs, thereby increasing the critical grain size $d_{c}$ (in accord with the results of our model) and decreasing hardness. If this is so, it is logical to assume that, similar to the case of nanocrystalline metals and alloys, the inverse Hall-Petch behavior in nanocrystalline ceramics can be suppressed through a thermal treatment or doping by impurities, which decrease GB energy and hinder GB sliding.

To summarize, dislocation emission from triple junctions of GBs can explain the transition from the direct to the inverse Hall-Petch dependence in nanocrystalline ceramics as well as the low strain rate sensitivity of such ceramics documented in ref. [9]. The critical grain size for this transition depends on the fraction of triple junctions that can emit lattice or GB dislocation at a specified stress. In turn, the intensity of GB dislocation emission from triple junctions can depend on the structure and energy of GBs and their chemical composition. The observations [16,17] of direct Hall-Petch dependences down to very small grain sizes can be explained by assuming that the critical grain size for the transition from the direct to the inverse Hall-Petch dependence for the materials synthesized in refs. [16,17] is smaller than the minimum grain size of the specimens fabricated in these experiments.

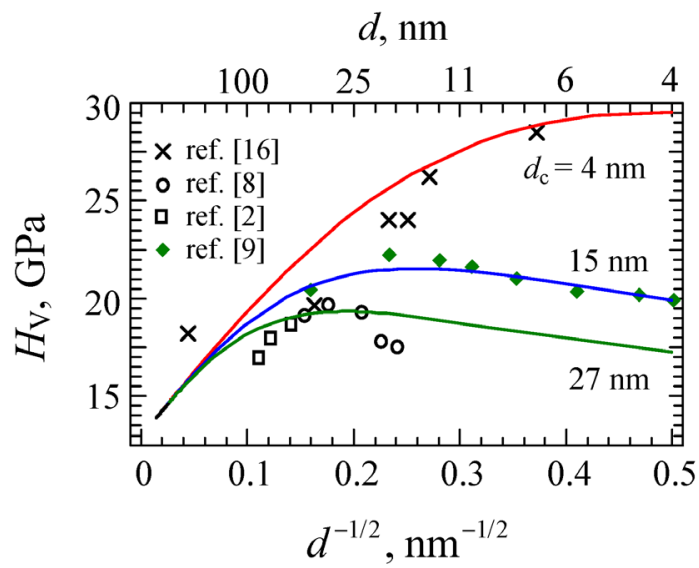

Fig. 2. (Color online) Calculated dependences of the microhardness $H_{V}$ of $\mathrm{MgAl}_{2} \mathrm{O}_{4}$ on the inverse of the square root of the grain size, $d^{-1 / 2}$. 
Aknowledgements. The authors acknowledge the support of the Russian Science Foundation (grant 18-19-00255).

\section{References}

1. J.A. Wollmershauser, B. N. Feigelson, E.P. Gorzkowski, C. T. Ellis, R. Goswami, S. B. Qadri, J. G. Tischler, F. J. Kub, R. K. Everett. Acta Mater. 69, 9 (2014). $\underline{\text { Crossref }}$

2. M. Sokol, M. Halabi, S. Kalabukhov, N. Frage. J. Europ. Ceram. Soc. 37, 755 (2017). Crossref

3. M. Sokol, S. Kalabukhov, R. Shneck, E. Zaretsky, N. Frage. J. Europ. Ceram. Soc. 37, 3417 (2017). Crossref

4. H. Wang, A. Sharma, A. Kvit, Q. Wei. J. Mater. Res. 16, 2733 (2001). Crossref

5. Y. Wang, J. Zhang and Y. Zhao. Nano Lett. 7, 2 (2007). Crossref

6. D. Ehre, R. Chaim. J. Mater. Sci. 43, 6139 (2008). Crossref

7. Z. B. Qi, P. Sun, F. P. Zhu, Z. C. Wang, D. L. Peng, C. H. Wu. Surf. Coat. Tech. 205, 3692 (2011). $\underline{\text { Crossref }}$

8. M. Sokol, M. Halabi, Y. Mordekovitz, S. Kalabukhov, S. Hayun, N. Frage. Scripta Mater. 139, 159 (2017). Crossref

9. H. Ryou, J.W. Drazin, K. J. Wahl, S.B. Qadri, E. P. Gorzkowski, B. N. Feigelson, J.A. Wollmershauser. ACS Nano. 12, 3083 (2018). Crossref

10. D. Guo, S. Song, R. Luo, W. A. Goddard, III, M. Chen, K. M. Reddy, Q. An. Phys. Rev. Lett. 121, 145504 (2018). Crossref

11. A. Bokov, J. B. Rodrigues Neto, F. Lin, R.H. R. Castro. J. Am. Ceram. Soc. 103, 2001 (2020). Crossref

12. L. Feng, A. Bokov, S.J. Dillon, R.H. R. Castro. J. Eur. Ceram. Soc. 40, 2050 (2020). Crossref

13. N.A. Gaida, N. Nishiyama, O. Beermann, U. Schürmann, A. Masuno, C. Giehl, K. Niwa, M. Hasegawa, S. Bhat, R. Farla, L. Kienle. Int. J. Ceramic Eng. Sci. 2, 76 (2020). Crossref

14. B. Ratzker, A. Wagner, M. Sokol, L. Meshi, S. Kalabukhov, N. Frage. Acta Mater. 183, 137 (2020). $\underline{\text { Crossref }}$

15. A. G. Sheinerman, M. Yu. Gutkin. Rev. Adv. Mater. Tech. 1, 46 (2019).
16. D. N.F. Mucho, J.W. Drazin, J. Mardinly, S. Dey, R.H.R. Castro. Mater. Lett. 186, 298 (2017). Crossref

17. C. Yang, A. Thron, R. H. R. Castro. J. Amer. Cer. Soc. 102, 6904 (2019). Crossref

18. Q. Feng, X. Song, X. Liu, S. Liang, H. Wang, Z. Nie. Nanotechnology. 28, 475709 (2017). Crossref

19. D. Guo, Q. An. Int. J. Plasticity. 121, 218 (2019). Crossref

20. B. Jiang, G. J. Weng. Int. J. Plasticity. 20, 2007 (2004). Crossref

21. A. G. Sheinerman, R. H. R. Castro, M. Yu. Gutkin. Mater. Lett. 260, 126886 (2020). Crossref

22. H. Conrad, J. Narayan. Scripta Mater. 42, 1025 (2000). Crossref

23. S. S. Quek, Z. H. Chooi, Z. Wu, Y. W. Zhang, D. J. Srolovitz. J. Mech. Phys. Sol. 88, 252 (2016). Crossref

24. G. I. Taylor. J. Inst. Met. 62, 307 (1938).

25. G. Mohanty, J.M. Wheeler, R. Raghavan, J. Wehrs, M. Hasegawa, S. Mischler, L. Philippe, J. Michler. Philos. Mag. 95, 1878 (2015). Crossref

26. Y. Kim, J. Lee, M.S. Yeom, J. W. Shin, H. Kim, Y. Cui, J. W. Kysar, J. Hone, Y. Jung, S. Jeon, S. M. Yan. Nature Commun. 4, 2114 (2013). Crossref

27. Z.C. Cordero, B.E. Knight, C.A. Schuh. Int. Mater. Reviews. 61, 495 (2016). Crossref

28. J.R. Cahoon, W.H. Broughton, A.R. Kutzak. Metall. Trans. 2, 1979 (1971). Crossref

29. A. J. Detor, C. A. Schuh. J. Mater. Res. 22, 3233 (2007). Crossref

30. J. Hu, Y. N. Shi, X. Sauvage, G. Sha, K. Lu. Science. 355, 1292 (2017). $\underline{\text { Crossref }}$

31. J. Gubicza. Adv. Eng. Mater. 22, 1900507 (2020). Crossref

32. A. M. Mavlyutov, T.A. Latynina, M.Yu. Murashkin, R.Z. Valiev, T.S. Orlova. Phys. Solid State. 59, 1970 (2017). Crossref

33. T. S. Orlova, N. V. Skiba, A.M. Mavlyutov, R.Z. Valiev, M. Yu. Murashkin, M. Yu. Gutkin. Rev. Adv. Mater. Sci. 57, 224 (2018). Crossref

34. M. Yu. Gutkin, T. A. Latynina, T. S. Orlova, N. V. Skiba. Phys. Solid State. 61, 1790 (2019). Crossref 